\title{
Understanding Clinical Importance of Frailty and Designing a Frailty Assessment Tool in Acute Hospitals
}

\author{
Oo $\mathrm{MT}^{1^{*}}$, Chan $\mathrm{YP}^{1}$, Chamberlain $\mathrm{H}^{2}$ and $\mathrm{Ho} \mathrm{SF}^{2}$ \\ ${ }^{1}$ Department of Geriatric Medicine, Good Hope Hospital, Sutton Coldfield, UK
}

${ }^{2}$ Department of Geriatric Medicine, Royal Shrewsbury Hospital, Shrewsbury, UK

*Correspondence author: Moe Thaw Oo, Department of Geriatric Medicine, Good Hope Hospital, Sutton Coldfield, UK, Tel: 0044-121 424 2000; E-mail: moethaw.oo@nhs.net

Rec date: Jul 18, 2014; Acc date: Sep 26, 2014; Pub date: Sep 28, 2014

Copyright: (c) 2014 Oo MT, et al. This is an open-access article distributed under the terms of the Creative Commons Attribution License, which permits unrestricted use, distribution, and reproduction in any medium, provided the original author and source are credited.

\begin{abstract}
Introduction: Managing the acute admission of older patients is a challenge in hospitals. Frail older people represent a larger proportion of acute admissions. Length of inpatient stay, inpatient mortality and the 90-day readmission rate are significant in this group of patients. In order to reduce the risks of adverse outcomes, frailty should be screened using an appropriate assessment tool and these patients must receive a Comprehensive Geriatric Assessment (CGA).
\end{abstract}

Methods: Based on current evidences a frailty screening tool was designed and developed. The tool was validated by means of testing the inter-observer and intra-observer reproducibility. The process of validation was conducted by independent observers who did not provide any contribution in developing this tool.

Results: A total of twenty patients were included. Ten patients were involved in testing the inter-observer reproducibility and eight out of total ten patients $(80 \%)$ were received the same frailty scores. The intra-observer reproducibility was evaluated in ten patients and ten out of total ten patients $(100 \%)$ obtained the same scores.

Conclusion: The results shown on testing the inter-observer and intra-observer reproducibility of the tool were satisfactory. Thus this tool can be considered as an applicable frailty screening tool in acute hospitals.

Keywords Frailty; The frailty screening tool; Acute older admissions

\section{Growth of Frailty Prevalence and Acute Geriatric Challenges in Hospitals}

In an older person, frailty can be generally defined as "a state of high vulnerability for adverse health outcomes, including disability, dependency, falls, need for long-term care and mortality" [1]. The United Nations have highlighted that the global elderly population will have grown by more than three times in the next five decades [2]. As the global ageing population grows, the prevalence of frailty will undoubtedly increase. Consequently the acute medical admissions of older people are getting increased in hospitals year by year. Nowadays managing elderly acute medical admissions is "a big challenge" in hospitals. For instance, in the UK acute hospitalisations of older people increased significantly by two-thirds from 2000 to 2010 [3]. Total hospital admissions of patients aged 75 and over were 2,308,480 in $1999 / 2000$, increasing to $3,837,990$ in $2009 / 2010$ [4]. Of these admissions, frail older people represented a larger proportion of acute admissions. The hospital admission statistics of the University Hospital of Leicester, England for example show that acute admissions of frail older people increase by approximately $5 \%$ per year [5].

About $50 \%$ of frail older patients who are rapidly discharged from acute medical care are re-admitted within the first 90 days from discharge [3]. Moreover, one-third of these frail elderly patients die within a year from discharge [6]. The length of inpatient stay and the mortality rate is also higher in frail than in the non-frail older patients [7]. A study relating to frail, older inpatients concluded that they had a higher risk of long inpatient stay and that a speedy discharge did not always serve them well [8]. All this evidence supports the argument that managing acute medical admissions of frail older people should be recognised as a major front-line issue in hospitals.

\section{A Comprehensive Approach to Geriatric Care and Frailty}

A comprehensive geriatric assessment (CGA) is the best approach in acute care for frail, older patients, as it yields overall positive outcomes for this group. A CGA is defined by a multidimensional diagnostic process, which focuses on determining a frail, older person's medical, psychological and functional capability in order to develop a coordinated and integrated plan for treatment and follow-up [6], [9]. A Cochrane review of CGA for the hospitalised elderly clearly stated that providing a CGA could increase a patient's survival and possibility of stay in their own residences by up to 12 months [9]. Overall, patients who received a CGA were discharged safely back to their own residences and stayed at their own homes for six weeks to 12 months on average [9]. Moreover, patients who were provided with integrated geriatric medical care gained better overall outcomes than those who were given general medical care [9]. Patients receiving a CGA in hospitals had less risk of being transferred to institutional care on discharge [9]. These evidences clearly show us that frail older patients should receive a CGA in hospitals without having any delay. 
In this circumstance a million-dollars valid question has been raised: "How is frailty assessed rapidly and appropriately among acute admissions of older people in hospitals?" More importantly acute hospitals are a busy clinical environment and a frailty screening tool, applicable in an acute clinical setting should therefore be a simple, clear, less time-consuming and user-friendly scale. It must cover major components of frailty. At this point the key predictors, triggering frailty in older people, should be clarified systematically based on the current evidences.

\section{Predictors of Frailty}

\section{Advanced age}

Many predictors are involved in the process of frailty in the elderly. There are inter-relations between frailty, disability, illnesses and advanced age. Illnesses can contribute as a triggering factor for frailty and a frail condition is aggravated by underlying disability and advanced age [10]. Advanced aging could also cause impaired homeostasis of the body that induces frailty [10]. The association between frailty and ageing with other influencing factors can be demonstrated as below (Figure 1).

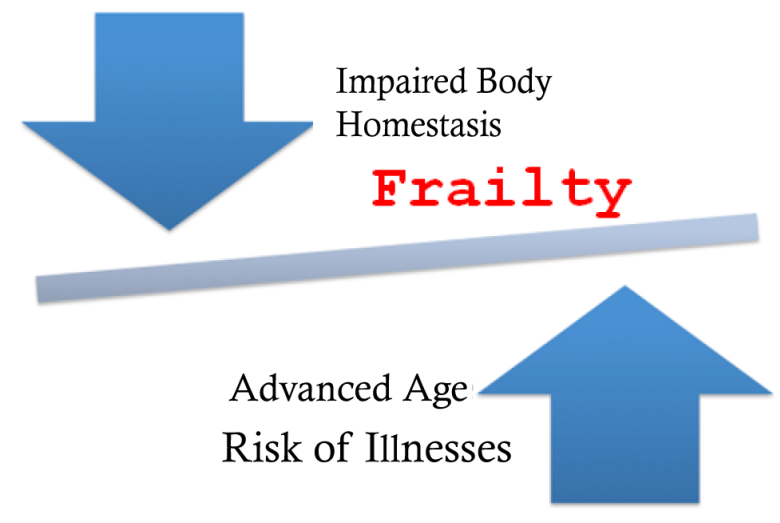

Figure 1: The link between frailty, aging, increased risk of illnesses and decreased body homeostasis.

\section{Frailty and falls}

Frailty and falls are closely related [11]. It is an important factor in causing falls and sometimes they can be the presenting clinical feature of the frail older person [11]. For instance, falls seem to be the result of a single factor such as syncope [11]. A cohort study of osteoporotic fractures, assessing the relationship between frailty, falls, fractures and mortality in older American women, found that frail older women had a higher risk of recurrent falls (multivariate odds ratio $1.38,95 \%$ confidence interval [CI], 1.02-1.88), hip fractures (95\% CI, 1.03-1.90) and death (95\% CI, 1.56-2.13) [12]. In addition, there is evidence that vitamin D deficiency (low 25-hydroxycholecalciferol) is common in frail older people and this deficiency increases the risk of frailty as well as falls [13].

Frail older people suffer significant loss of normal muscle capacity occurring as a result of impairments in the central and peripheral neurological functions of the body. Loss of muscle capacity, mainly in the limbs, causes poor balance and impaired gait [14]. Both balance and gait impairments are key factors that cause falls in the elderly $[11,14]$. Frailty can increase the severity of incidental fractures and mortality. A systematic review relating to frailty and fractures determined that the absolute risk of incidental fractures and mortality are significantly higher in frail people [15].

\section{Frailty and cognitive impairment}

According to an observational cohort study of community-dwelling older people in the US, frailty increases the risk of mild cognitive impairment (MCI) as well as its severity [16]. Similar results were found in an epidemiological study of older Mexican Americans, where a strong relationship between frailty and cognitive dysfunction in older people was found [17]. Cognitive impairment in frail older people can present with altered executive function, slow gait speed and weak grip strength [17]. Older age, poor education and living alone with multiple co-morbidities can exacerbate cognitive decline and frailty [17]. Though more research is needed to identify clear explanations for the relationship between frailty and cognitive impairment, it has been established that frail older people have a higher risk of cognitive impairment compared to the non-frail elderly [17].

Frailty is also associated with the incidence of Alzheimer's dementia [18]. The psychological stressors of frailty, such as depression and loneliness, could be closely linked with the risk of Alzheimer's dementia. The same influencing factors causing frailty could lead to the development of Alzheimer's dementia [18].

\section{Frailty and care home residents}

In the UK about $70 \%$ of care home places are occupied by frail older people with multiple co-morbidities, such as dementia and stroke [19]. This survey also found that a large proportion of care home residents suffered long-term cognitive impairment and incontinence [19]. Frailty is the second most common reason for long-term care home placement in older people [19]. About $22 \%$ of total nursing home residents and $34 \%$ of total residential home residents have frailty. Frailty is therefore recognised as a significant contributing factor for institutionalisation in the UK's older people [19]. The Health Survey for England (2000) also showed that about $4 \%$ of the total middle aged and older population (age 65 years and above) were care home residents. In this group of people, about $75 \%$ were severely disabled [20]. Care home residents often need frequent hospitalisations and this group represented the majority of elderly acute admissions in hospitals $[19,20]$.

\section{Frailty and reduced mobility}

The cycle of frailty could be induced and started by a lack of physical activity, unhealthy lifestyles the ageing process [10]. These factors could greatly restrict the physical activities of the elderly and deregulate normal physiological systems of the body [10]. For example, when people grow older, normal cardiovascular and musculoskeletal reserves of the body gradually decline and this negatively affects the cardio-pulmonary functions as well as mobility [21]. Sarcopenia, a condition in which loss of skeletal muscle mass occurs secondary to aging, is one of the major causes of reduced physical activity as well as mobility in the elderly [22]. Restricted physical functions increase the chance of sarcopenia and all these conditions are perpetuated in a vicious cycle [22]. Moreover, these changes could reduce the resting metabolic rate, total energy 
expenditure and energy output of the body, causing a state of undernourishment $[10,23]$. All of these factors are closely inter-related and occur together, leading to the frailty state $[10,23]$.

\section{Designing a Frailty Screening Tool Applicable in Acute Hospitals}

As frailty is a complex geriatric syndrome, screening for frailty is not an easy task. The nature of frailty is a dynamic condition, not a static state [24]. This also makes it more difficult to establish a comprehensive assessment scale for frailty. However, the clinical significance of frailty indicates that it should be screened for. There are different frailty assessment tools designed, implemented and delivered to different target elderly populations in different countries. Most of these tools are generally applied for research purposes rather than for clinical practice. Although many assessment tools and screening scales have emerged over the past decade, no single scale is universally accepted [24]. In terms of reliability and clinical effectiveness, many variations such as defining the criteria of frailty, exist in the different frailty screening scales and assessment tools [24]. For instance cognitive impairment is not in the Fried Frailty Phenotype whereas delirium and dementia are seen as key criteria in the Canadian Frailty Index proposed by Rockwood and colleagues [24].

A systematic review of a variety of frailty screening tools mentioned that no single best intervention can be validated thus far in the assessment of frailty in hospitalised older people [24]. Therefore, designing a screening tool for frailty should directly reflect and relate to the purposes of identifying frailty in target older populations [24]. A frailty tool designed for a research study or a randomised controlled trial might be different from that designed for daily clinical use [24]. There is therefore no hard and fast rule in designing a screening tool for frailty [24]. It should be set up appropriately with recognition of local needs and proposed applications. In other words, it should be designed and devised based on the application [24]. Evidence-based major criteria of frailty should be included in order to assess frailty appropriately [24].

We designed and developed a frailty screening tool by taking into account the following key features

\section{Design}

- Must cover major components of frailty

- Must be simple and clear

- Must be useful for clinical practice

- Must be applicable as a quick assessment in busy clinical settings

- Must be easily used by any member of the acute admission team

\section{Criteria}

- Age (75 and over)

- Fall with any injury or fracture (excluding fracture of the neck of the femur)

- Dementia or delirium

- Care home residence

- Reduced or lack of mobility over 24 hours 24 hours

If any acute elderly inpatient meets at least three out of five of these criteria, this patient should be diagnosed as having frailty, indicating a need for a CGA.
The background rationale for establishing these criteria in the frailty screening tool are:

- Aging should be seen as a key parameter influencing frailty in older people (seen details in Section 3.1).

- Fall and fragility fractures are directly proportionate to risk of fragility (seen details in section 3.2). However, fracture of the neck of the femur should be excluded as there is a separate assessment and care pathway for older people with this in hospitals.

- Any kind of cognitive impairment either acute or chronic closely links with frailty in old age (seen details in section 3.3).

- Prevalence of frailty is very common in institutional-dwelling older people (seen details in section 3.4).

- Reduced mobility or physical activities co-relates with frailty in the elderly (seen details in section 3.5).

\section{Critique of the Frailty Screening Tool}

The strengths and weaknesses of our frailty screening tool are evaluated as follows:

\section{Strengths of the tool}

A reliable measurement scale has a good level of precision and accuracy [25]. From the perspective of clinical research methodologies, precision is defined by the degree to which the same results are gained in repetitive use, and accuracy is described as the degree to which the collected results actually represent what they are supposed to represent [25]. Both precision and accuracy play an important role in any measurement scale, as precision promotes the detection ability of the scale and accuracy enhances its validity [25]. To maintain these qualities of measurement this frailty screening tool consists of standardised fixed criteria, apart from: "reduced or lack of mobility over 24 hours" [25]. The validity of these fixed criteria should therefore be recognised as constant, applying in any group of patients in any clinical circumstance.

The inter-observer and intra-observer reproducibility of the tool were tested from 1st March to 14th March 2012 in Good Hope Hospital, Sutton Coldfield, and England as follows:

Ten patients, aged 75 years and above, acutely and medically admitted to the hospital were randomly selected. Using the frailty screening tool, frailty was assessed among these patients. The rate of inter-observer reproducibility was mainly tested. Two different paired observers (two different grades of doctor, a doctor and a physiotherapist) assessed the same patient with the tool [25]. Eight out of total ten patients $(80 \%)$ were given the same frailty scores, whereas the other two patients (20\%) were given different scores by only 'one mark', relating to the criterion "reduced or lack of mobility in last 24 hours".

This parameter may be scored by direct observation of patients, or self-reporting by patients or carers, thus causing either observer or participant bias. However, these potential biases are only associated with one out of five parameters on the whole scale, and therefore the overall validity of the scale or the final frailty outcome resulting from the scale should not be altered significantly. The frailty assessment scores that two different paired observers had marked are summarised in the Table 1 below. In order to evaluate intra-observer reproducibility of the scale, frailty was assessed among ten patients in different clinical settings. In this assessment one observer (a doctor) assessed the same patient in two different clinical settings; the 
emergency department (A\&E) and the acute medical admission unit (AMU).

\begin{tabular}{|l|l|l|}
\hline & One doctor and therapist & Two different doctors \\
\hline Patient 1 & Frailty score $3 / 5$ & Frailty score $3 / 5$ \\
\hline Patient 2 & Frailty score $4 / 5$ & Frailty score $3 / 5$ \\
\hline Patient 3 & Frailty score $4 / 5$ & Frailty score $4 / 5$ \\
\hline Patient 4 & Frailty score 4/5 & Frailty score $4 / 5$ \\
\hline Patient 5 & Frailty score $5 / 5$ & Frailty score $4 / 5$ \\
\hline Patient 6 & Frailty score $3 / 5$ & Frailty score $3 / 5$ \\
\hline Patient 7 & Frailty score $2 / 5$ & Frailty score $2 / 5$ \\
\hline Patient 8 & Frailty score $5 / 5$ & Frailty score $5 / 5$ \\
\hline Patient 9 & Frailty score $2 / 5$ & Frailty score $2 / 5$ \\
\hline Patient 10 & Frailty score 4/5 & Frailty score $4 / 5$ \\
\hline
\end{tabular}

Table 1: Frailty assessment scores from different paired observers.

(NB: Patients 2 and 5 highlighted in red received different scores on frailty assessment).

Five out of a total of five patients (100\%) received the same frailty score, proving that different clinical settings did not change the validity of the screening tool. In other words intra-observer reproducibility of this frailty screening scale is satisfactory. The frailty assessment scores that the observer marked in different clinical settings are listed in the Table 2 below:

\begin{tabular}{|l|l|l|}
\hline & A and $\mathrm{E}$ & AMU \\
\hline Patient 1 & Frailty score $3 / 5$ & Frailty score $3 / 5$ \\
\hline Patient 2 & Frailty score $3 / 5$ & Frailty score $3 / 5$ \\
\hline Patient 3 & Frailty score $4 / 5$ & Frailty score $4 / 5$ \\
\hline Patient 4 & Frailty score $3 / 5$ & Frailty score $3 / 5$ \\
\hline Patient 5 & Frailty score $5 / 5$ & Frailty score $5 / 5$ \\
\hline Patient 6 & Frailty score $5 / 5$ & Frailty score $5 / 5$ \\
\hline Patient 7 & Frailty score $4 / 5$ & Frailty score $4 / 5$ \\
\hline Patient 8 & Frailty score $3 / 5$ & Frailty score $3 / 5$ \\
\hline Patient 9 & Frailty score $2 / 5$ & Frailty score $2 / 5$ \\
\hline Patient 10 & Frailty score $2 / 5$ & Frailty score $2 / 5$ \\
\hline
\end{tabular}

Table 2: Frailty assessment scores from one observer at two different clinical units.

All observers involved did not contribute in developing the tool. They were all independent observers. Risk of researcher bias was therefore minimised in the process of validation.

\section{Weaknesses of the tool}

"Reduced or lack of mobility over 24 hours" is a subjective factor in identifying frailty and it could vary from patient to patient. It impacts on the validity of this specific factor. Only twenty patients were involved in testing inter- and intra-observer reproducibility. The small sample size could increase the chance of standard error $[25,26]$ and affect the precision of this measurement tool [25].

\section{Conclusion}

As the acute admissions of older patients and prevalence of frailty are high, it is imperative to conduct frailty screening in daily acute elderly care practice for applying a CGA rapidly and effectively. Our frailty screening tool showed satisfactory outcomes in term of testing its inter-observer and intra-observer reproducibility. Thus it is generally justified that this tool can be applicable in acute clinical settings. Further multi-centered validation should be conducted to prove of its generalizability.

\section{References}

1. Fried LP, Tangen CM, Walston J (2001) Frailty in older adults, evidence for a phenotype. Journal of Gerontology 56: 46-157.

2. Department of Economic and Social Affairs, United Nations. World population aging 1950-2050.

3. The NHS Information Centre for Health and Social Care. Report on NHS hospitals activities 2010 .

4. The NHS Information Centre for Health and Social Care. Hospital episode statistics: admitted patient care 2009.

5. Woodard J, Gladman J, Conroy S (2010) Frail older people at the interface. Age and Ageing 39: 36.

6. Conroy S, Stevens T, Parker SG (2011) A systematic review of comprehensive geriatric assessment to improve outcomes for frail older people being rapidly discharged from acute hospital: 'interface geriatrics'. Age and aging 40:436-443.

7. Conroy S, Carver J, Johnston K (2010) The Acute Frailty Unit - a novel approach to managing frail older people in the AMU. Age and Ageing 39: 40.

8. Woodard J, Gladman J, Conroy S (2009) Frail older people at the interface. JNHA 13: S308.

9. Ellis G, Whitehead MA, O'Neill D (2011) Comprehensive geriatric assessment for older adults admitted to hospital. Cochrane Database Syst Rev 7: CD006211.

10. Lang PO, Michel JP, Zekry D (2009) Frailty syndrome: transitional state in dynamic process. Journal of Gerontology 55: 539-549.

11. Nowak A, Hubbard RE (2009) Falls and frailty: lessons from complex systems. J R Soc Med 102: 98-102.

12. Ensrud KE, Stone KL (2007) Frailty and risk of falls, fracture, and mortality in older women: the study of osteoporotic fractures. J Gerontol A Biol Sci Med Sci 62:744-751.

13. Puts MT, Visser M, Twisk JW (2005) Endocrine and inflammatory markers as predictors of frailty. Clin Endocrinol 63:403-411.

14. Buchman AS, Schneider JA, Wilson RS (2006) Body mass index in older persons is associated with Alzheimer disease pathology. Neurology 67:1949-1954.

15. van den Bergh JP, van Geel TA, Geusens PP (2012) Osteoporosis, frailty and fracture: implications for case finding and therapy. Nat Rev Rheumatol 17: 163-172.

16. Boyle PA, Buchman AS, Wilson RS (2010) Physical frailty is associated with incident mild cognitive impairment in community-based older persons. J Am Geriatr Soc 58: 248-255.

17. Samper-Ternent R, Al Snih S, Raji MA (2008) Relationship between frailty and cognitive decline in older Mexican Americans. J Am Geriatr Soc 56: 1845-1852.

18. Buchman AS, Boyle PA, Wilson RS (2007) Frailty is associated with incident Alzheimer's disease and cognitive decline in the elderly. Psychosomatic Medicine 69:483-489. 
Citation: $\quad$ Oo MT, Chan YP, Chamberlain H, Ho SF (2014) Understanding Clinical Importance of Frailty and Designing a Frailty Assessment Tool in Acute Hospitals. J Gerontol Geriat Res 3: 1000176. doi:10.4172/2167-7182.1000176

Page 5 of 5

19. Bowman C, Whistler J, Ellerby M (2004) A national census of care home residents. Age and Ageing 33:561-566.

20. Bajekal M (2002) Health survey for England 2000: characteristics of care homes and their residents. London: The Stationery Office.

21. Flegg JL, Lakatta EG (1988) Role of muscle loss in the age-related associated reduction in VO2 max. J Appl Physiol 65: 1147-1151.

22. Morley JE (2008) Diabetes, sarcopenia and frailty. Clin Geriatr Med 24: 455-469.

23. Fried LP, Tangen CM, Walston J (2001) Cardiovascular Health Study Collaborative Research Group: frailty in older adults: evidence for a phenotype. J Gerontol A Biol Sci Med Sci 2001; 56: 146-156.
24. Sternberg SA, Wershof Schwartz A, Karanananthan S (2011) The identification of frailty: a systematic literature review. JAGS 59:21292138.

25. Hulley SB, Cummings SR, Browner WS (2010) Designing clinical research: an epidemiologic approach. 3rd edition. Philadelphia: Lippincott Williams and Wilkins Publishing 38-45.

26. Kalra P (2006) Essential revision notes for MRCP. Revised 1st edition. Knutsford, Cheshire: Pastest Ltd Publishing 648-653. 